\title{
Characterising rhodopsin-arrestin interactions with the fragment molecular orbital (FMO) method
}

Alexander Heifetz ${ }^{1 *}$ and Andrea Townsend-Nicholson ${ }^{2}$

${ }^{1}$ Evotec (UK) Ltd., 114 Innovation Drive, Milton Park, Abingdon, Oxfordshire OX14 4RZ, United Kingdom

${ }^{2}$ Research Department of Structural \& Molecular Biology, Division of Biosciences, University College London, London, WC1E 6BT, United Kingdom

\section{Keywords:}

Arrestin, Rhodopsin, G protein-coupled receptors, GPCR, chemical interactions, pairinteraction energy, QM, Quantum Mechanics, FMO, Fragment Molecular Orbitals method, biased ligands, signaling. 


\section{Abstract}

Arrestin binding to $\mathrm{G}$ protein-coupled receptors (GPCRs) plays a vital role in receptor signalling. Recently, the crystal structure of rhodopsin bound to activated visual arrestin was resolved using XFEL (X-ray free electron laser). However, even with the crystal structure in hand, our ability to understand GPCR-arrestin binding is limited by the availability of accurate tools to explore receptor-arrestin interactions. We applied Fragment Molecular Orbital Method (FMO) to explore the interactions formed between the residues of rhodopsin and arrestin. FMO enables ab initio approaches to be applied to systems that conventional quantummechanical (QM) methods would be too compute-expensive. The FMO calculations detected 35 significant interactions involved in rhodopsin-arrestin binding formed by 25 residues of rhodopsin and 28 residues of arrestin. Two major regions of interaction were identified: at the C-terminal tail of rhodopsin (D330-S343) and where the 'finger loop' (G69-T79) of arrestin that directly inserts into rhodopsin active core. Out of these 35 interactions, 23 were mainly electrostatic and 12 hydrophobic in nature. 


\section{Introduction}

1.1. G protein-coupled receptors (GPCRs) are a large family of membrane receptors that mediates transmembrane signalling through $\mathrm{G}$ proteins and arrestins [ref]. Arrestins are a small family of proteins with four human isoforms [1] that play a key role in homologous desensitization of GPCRs, as well as regulating several other vital cellular signalling pathways [1-3]. Remarkably, only two arrestins regulate the signalling of $>800$ GPCRs [2]. Several G-protein-independent pathways have been identified, with the $\beta$-arrestin-dependent signalling pathway the best characterised, to date [4].

1.2. GPCRs exist in multiple, distinct conformational states and form population ensembles comprised of several of these different active and inactive states. Ligands tend to bind to one of these states, increasing its population, which in turn activates (or deactivates) a particular signalling pathway via an effector (e.g. G proteins or $\beta$-arrestins). Ligands that preferentially activate a specific GPCR signalling pathways are known as 'biased ligands'. Further study is required to determine how, at the atomic level, GPCRs adopt specific conformations that lead to a preference for a particular effector, whether a $G$ protein or an arrestin.

1.3. Recently, the crystal structure of rhodopsin bound to activated visual arrestin has been solved using XFEL (X-ray free electron laser) (PDB code 5W0P) [5] (Figure 1A). However, even with the crystal structure in hand, the "visual inspection" and force field-based molecular mechanics (MM) calculations often used for structural exploration cannot always explain the full complexity of the molecular interactions between GPCR and arrestin [6].

1.4. Fragment Molecular Orbital Method (FMO) [7-9] readily brings the power of QM to molecular biochemical research and drug discovery. FMO provides insights into the 
chemical nature of interactions that are normally difficult to detect with non-QM methods. The FMO approach has a proven track record in the deep analysis of crystal or modelled GPCR structures and in characterizing the interactions between receptors and ligands [10]. FMO calculations are reasonably straightforward to set up and can be performed on relatively small PC clusters within an acceptable timeframe. FMO analysis can result in two considerable benefits: (a) complex QM theories are condensed into four simple and intuitively clear quantities, and (b) calculations become much faster than those performed using traditional QM approaches [11].

1.5. FMO works by partitioning a large system such as a protein into small fragments, for example, each residue in a protein can be represented as a fragment (Figure 1a). By performing QM calculations on fragments, one can make the computational cost scaling with respect to system size nearly linear. One of the key features of the FMO approach is that it can provide a list of the interactions formed between the fragments of the system under study, yielding a chemically-intuitive breakdown of these interactions (Figure 1b) [12].

$<$ Figure 1 here $>$

Figure 1. (a) Crystal structure of the rhodopsin-arrestin complex (PDB code 5W0P) [5]. (b) Details of each of the PIE terms that are computed using the FMO workflow [12]. The electrostatic component arises from the Coulomb interaction between polarized charge distributions of fragments. The exchange repulsion term is derived from the interaction between fragments situated in close proximity and is always repulsive; it is due to the Pauli repulsion and is related to the overlap of two occupied orbitals. The charge transfer term arises from the interaction between occupied orbitals of a donor and unoccupied orbitals of an acceptor. The dispersion arises as the interaction between instantaneous dipole moments of two fragments, it is hydrophobic (non-polar) in nature and is obtained in PIEDA from the correlation energy of electrons.

1.6. The pair interaction energy (PIE) between any two fragments calculated by FMO is the sum of four energy terms: electrostatic, exchange-repulsion, charge transfer and 
dispersion, provided by pair interaction energy decomposition analysis (PIEDA) [13] - see Figure 1b. The electrostatic and charge transfer terms are predominant in saltbridge, hydrogen bond and polar interactions, whilst the dispersion term generally corresponds to interactions that are predominantly hydrophobic in nature. The role of hydrophobic interactions is integral for biomolecular recognition but there is still no reliable predictive method for its quantification [6]. The exchange-repulsion term is a high level QM term which quantifies the repulsion between electrons [12].

1.7. FMO is an extensively validated method for the structural exploration [14] of large biological systems. In this report, we apply it to explore interactions between residues of the rhodopsin-arrestin complex (PDB code 5W0P, [5]).

\section{Methods}

2.1. For FMO analysis of the rhodopsin-arrestin structure, we used FMO as implemented in General Atomic and Molecular Electronic Structure System (GAMESS). In FMO calculations, a large biological system is partitioned into fragments (Figure 1) [7, 8]. For example, each residue within a protein can be represented by a fragment. The detailed description of the FMO strategy and methodology can be found in the published reports $[7,8,13]$, including a detailed mathematical formulation that are beyond the scope of this chapter and described in detail in chapter 11 of this book. 
2.2. We used the MP2 method (Second Order Møller-Plesset perturbation theory [15]) with the $6-31 G^{*}$ basis set. This basis set is most commonly used and is often considered the best compromise between speed and accuracy [16]. Residues and water molecules within a radius of $\leq 4.5 \AA$ around the ligand atoms were included in the FMO calculations, since previous work demonstrated [10] that including these atoms significantly increases the speed of the calculation without compromising the results. FMO can be implemented with a polarizable continuum solvent model (PCM), which ameliorates the effect of charged residues around the system of interest [17].

2.3. The residues of arrestin are numbered as for the rhodopsin-arrestin crystal structure (PDB code 5W0P) [5]. Arrestin elements are mapped based on the recent publication of Gurevich et al [18] (Figure 2).

$<$ Figure 2 here $>$

Figure 2: Sequence of arrestin as extracted from rhodopsin-arrestin crystal structure (PDB code 5WOP) [5]. Arrestin elements are coloured as follows: Green: 'finger loop', G69-T79; Yellow: '157-loop', D156-I165; Cyan: 'C-loop', V249-S253 and Pink: 'back loop', R319-M322; 


\section{Notes}

3.1. Despite the extensive efforts described in the literature $[1-3,5,18]$, the specific molecular interactions, in terms of interaction energy and chemical nature, between rhodopsin and arrestin have not yet been characterised. We applied the FMO method to investigate the interactions between rhodopsin and arrestin as evidenced in the crystal structure 5W0P [5].

\section{$<$ Figure 3 here $>$}

Figure 3. (a) Network of 35 conserved inter-TM interactions involved in rhodopsin-arrestin binding for crystal structure 5WOP [5], formed by 25 residues of rhodopsin and 28 residues of arrestin. The ellipsoids represent residues. The lines between a pair of circles indicate the presence of interaction energy and the thickness of the line is proportional to the size of the PIE. Only interactions with absolute value of $>3.0 \mathrm{kcal} / \mathrm{mol}$ are shown. Lines are coloured according to their chemical factor $\left(f=E^{\text {electrostatic }} / E^{\text {dispersion }}+E^{\text {electrostatic }}\right)$ : from $100 \%$ electrostatic (yellow) to $100 \%$ hydrophobic (dark blue). (b) The GPCR is shown in ribbon form and the location of the 35 interactions on the surface of arrestin is highlighted in magenta.

3.2. The FMO calculations detected 35 significant interactions involved in rhodopsinarrestin binding (Figure 3a); these were formed by 25 residues of rhodopsin and 28 residues of arrestin. Of these 35 interactions, 23 had mainly electrostatic nature and 12 hydrophobic (Figure 3a). Two major regions of interactions were identified: the Cterminal tail of rhodopsin (D330-S343) and the finger loop of arrestin (Figure 3b) that directly inserts into rhodopsin active core.

$<$ Figure 4 here $>$

Figure 4. Six salt bridges formed between phosphorylated residues Tpo336 and Sep338 of rhodopsin and arrestin 
3.3. FMO detected that the C-tail of rhodopsin forms 16 interactions with arrestin. This FMO result is in agreement with a previous report [5] suggesting that the C-terminal tail of rhodopsin plays a role as one of the anchor points for rhodopsin-arrestin binding. FMO detected that the phosphorylated residues pT336 (Tpo336) and pS338 (Sep338) of rhodopsin form an extensive network of salt bridges (Figure 4) with the positively charged residues of arrestin: Thr336 forms three salt bridges with Lys16, Arg19 and Arg172 while Ser338 forms three salt bridges with Lys16, Arg30 and Arg301.

3.4. FMO has highlighted the significant role of the 'finger-loop' of arrestin (Gly69-Thr79, Figure 2) in rhodopsin binding, with six out of the ten residues of this helical loop involved in rhodopsin binding (Finger 3a). Some of these residues form more than one interaction, for example, Met76 of arrestin forms interactions with both Gln312 and Phe313 of the C-terminus of rhodopsin and Leu78 forms hydrophobic interactions with both Val250 and Met253 of rhodopsin's TM6 domain. The molecular basis underpinning the role of the 'finger-loop' of arrestin in rhodopsin binding as established by FMO rationalizes the experimental observation that deletion of Gly69 at the beginning of the finger-loop dramatically reduced binding of arresting to all GPCRs [18], reinforcing the critical effect of this residue on the stability of the secondary structure of arrestin's finger-loop.

3.5. FMO has also emphasized the significant contribution of underappreciated interactions in the rhodopsin-arresting complex, including hydrophobic interactions and non-classical hydrogen bonds, with 20 out of all of 35 interaction being of this type (Finger 5). 12 of the 35 interactions were of a hydrophobic nature (Figure 3a). Additional examples of underappreciated interactions include those formed by rhodopsin's Phe146 (located on intracellular loop 2). Phe146 forms interactions with 4 different arrestin residues (Figure 5a) as follows: a hydrogen bond with the $\mathrm{NH}$ backbone of Lys142, a hydrophobic interaction with Pro35, a $\mathrm{CH}-\pi$ interaction with 
Cys144 and a non-classical hydrogen bond with the backbone carbonyl of Leu133.

Other examples of underappreciated interactions are illustrated in Figure 5a-5d.

$<$ Figure 5 here>

Figure 5. (a) Phe146 (rhodopsin) forms interactions with 4 different arrestin residues: a hydrogen bond with the $\mathrm{NH}$ backbone of Lys142,a hydrophobic interaction with Pro35, a $\mathrm{CH}-\pi$ interaction with Cys144 and a non-classical hydrogen bond with the backbone carbonyl of Leu133. (b) Backbone atoms of Glu341 (rhodopsin) form two hydrogen bonds with the backbone atoms of lle13 (arrestin) while the side-chain of Glu341 forms salt bridge Lys111 (c) $\mathrm{CH}-\pi$ interaction of Met143 (rhodopsin) with Tyr256 (arrestin) and (d) Nonclassical hydrogen bond formed between Val139 (rhodopsin) and Gly77 (arrestin).

3.6. These FMO results are not only in agreement with the published data $[1,2,5,18]$, they have provided additional quantitative information regarding the energetic strengths of these interactions and their chemical nature. FMO also provided information regarding underappreciated interactions that were not previously detected by any other method. These FMO structure-function studies reveal several different ways of increasing the capacity of arrestins to quench GPCR signaling, facilitating the development of enhanced arrestins with the potential to serve as tools for gene therapy of disorders associated with excessive signaling of mutant GPCRs [2].

3.7. FMO is a valuable resource that can be applied to aid further understanding of GPCR function. The regular application of FMO to GPCR studies can be used to expedite the generation of more effective GPCR-biased drugs $[19,20]$. This utility of FMO is not restricted to GPCRs, however, as it can be applied to the structural exploration of other protein families and biosystems. 


\section{ACKNOWLEDGMENT}

A.H. and A.T.N. would like to acknowledge the support of EU H2020 CompBioMed project (http://www.compbiomed.eu/, 675451). 


\section{References}

1. Chen Q, Iverson TM, Gurevich VV, (2018), Structural Basis of Arrestin-Dependent Signal

Transduction, Trends Biochem Sci. 43: 412-423.

2. Gurevich VV, Gurevich EV, (2019), Arrestin mutations: Some cause diseases, others promise cure, Prog Mol Biol Transl Sci. 161: 29-45.

3. Gurevich VV, Gurevich EV, (2013), Structural determinants of arrestin functions, Prog Mol Biol Transl Sci. 118: 57-92.

4. Tan L, Yan W, McCorvy JD, Cheng J, (2018), Biased Ligands of G Protein-Coupled Receptors (GPCRs): Structure-Functional Selectivity Relationships (SFSRs) and Therapeutic Potential, J Med Chem. 61: 9841-9878.

5. Zhou XE, He Y, de Waal PW, Gao X, Kang Y, Van Eps N, Yin Y, Pal K, Goswami D, White TA, Barty A, Latorraca NR, Chapman HN, Hubbell WL, Dror RO, Stevens RC, Cherezov V, Gurevich VV, Griffin PR, Ernst OP, Melcher K, Xu HE, (2017), Identification of Phosphorylation Codes for Arrestin Recruitment by G Protein-Coupled Receptors, Cell. 170: 457-469.e413.

6. Bissantz C, Kuhn B, Stahl M, (2010), A medicinal chemist's guide to molecular interactions, J Med Chem. 53: 5061-5084.

7. Fedorov DG, Nagata T, Kitaura K, (2012), Exploring chemistry with the fragment molecular orbital method, Phys Chem Chem Phys. 14: 7562-7577.

8. Fedorov DG, Kitaura K, (2007), Extending the power of quantum chemistry to large systems with the fragment molecular orbital method, J Phys Chem A. 111: 6904-6914.

9. Kitaura K, Ikeo E, Asada T, Nakano T, Uebayasi M, (1999), Fragment molecular orbital method: an approximate computational method for large molecules, Chemical Physics Letters. 313: 701-706.

10. Heifetz A, Chudyk El, Gleave L, Aldeghi M, Cherezov V, Fedorov DG, Biggin PC, Bodkin MJ, (2016), The Fragment Molecular Orbital Method Reveals New Insight into the Chemical Nature of GPCR-Ligand Interactions, J Chem Inf Model. 56: 159-172.

11. Alexeev Y, Mazanetz MP, Ichihara O, Fedorov DG, (2012), GAMESS as a free quantummechanical platform for drug research, Curr Top Med Chem. 12: 2013-2033.

12. Phipps MJ, Fox T, Tautermann CS, Skylaris CK, (2015), Energy decomposition analysis approaches and their evaluation on prototypical protein-drug interaction patterns, Chem Soc Rev. 44: 3177-3211.

13. Fedorov DG, Kitaura K, (2007), Pair interaction energy decomposition analysis, J Comput Chem. 28: 222-237.

14. Chudyk EI, Sarrat L, Aldeghi M, Fedorov DG, Bodkin MJ, James T, Southey M, Robinson R, Morao I, Heifetz A, (2018), Exploring GPCR-Ligand Interactions with the Fragment Molecular Orbital (FMO) Method, Methods Mol Biol. 1705: 179-195.

15. Fedorov DG, Kitaura K, (2004), Second order Moller-Plesset perturbation theory based upon the fragment molecular orbital method, J Chem Phys. 121: 2483-2490.

16. Mazanetz MP, Ichihara O, Law RJ, Whittaker M, (2011), Prediction of cyclin-dependent kinase 2 inhibitor potency using the fragment molecular orbital method, J Cheminform. 3: 2.

17. Li H, Fedorov DG, Nagata T, Kitaura K, Jensen JH, Gordon MS, (2010), Energy gradients in combined fragment molecular orbital and polarizable continuum model (FMO/PCM) calculation, $\mathrm{J}$ Comput Chem. 31: 778-790.

18. Zheng C, Tholen J, Gurevich VV, (2019), Critical role of the finger loop in arrestin binding to the receptors, PLoS One. 14: e0213792.

19. Sloop KW, Emmerson PJ, Statnick MA, Willard FS, (2018), The current state of GPCR-based drug discovery to treat metabolic disease, $\mathrm{Br} \mathrm{J}$ Pharmacol.

20. Hauser AS, Attwood MM, Rask-Andersen M, Schioth HB, Gloriam DE, (2017), Trends in GPCR drug discovery: new agents, targets and indications, Nat Rev Drug Discov. 16: 829-842. 